\title{
Professor Renting Li's Combination of Internal and External Treatment of Traditional Chinese Medicine in Treating Radiation Enteritis
}

\author{
Nannan $\mathrm{Yu}^{1^{*}}$, Renting $\mathrm{Li}^{2}$ \\ ${ }^{1}$ Shaanxi University of Chinese Medicine, Xianyang 712000, Shaanxi Province, China \\ ${ }^{2}$ The Affiliated Hospital of Shaanxi University of Chinese Medicine, Xianyang 712000, Shaanxi Province, China \\ *Corresponding author: Nannan Yu, 1164063360@qq.com
}

\begin{abstract}
Radiotherapy is a common treatment for abdominal and pelvic malignancies with bone metastases. However, some patients develop radiation enteritis (RE) during the treatment or 2 months or more after the treatment, which seriously affects their quality of life. At present, the curative effect of western medicine is poor. Professor Renting Li believes that radiation kills tumor cells but at the same time, it damages the healthy Qi of the human body. Radiation causes heat and poison to accumulate in the intestinal tract, consumes and hurts Yin fluid, burns the vein, leads to deficiency of healthy Qi and poor detoxification, as well as cause blood stasis after a period of time. All these result in the combination of heat, blood stasis, and poison, manifesting as abdominal pain, diarrhea, mucus in stools, purulent and bloody stools, etc. Therefore, Professor Renting Li proposed the use of traditional Chinese medicine retention enema combined with oral traditional Chinese medicine to reduce symptoms, remove toxins, and improve the quality of life of patients.
\end{abstract}

Keywords: Radiation enteritis; Traditional Chinese medicine; Internal and external treatment of traditional Chinese medicine

Publication date: November 2021; Online publication: November 30, 2021

\section{Introduction}

Radiation enteritis (RE) is a common complication after radiotherapy for abdominal and pelvic malignancies. According to relevant research reports, the incidence rate of $\mathrm{RE}$ is about 5\% 55\% in all patients ${ }^{[1]}$. Radiosensitive cells are generally undifferentiated; they divide rapidly and have high metabolic activity ${ }^{[2-3]}$. Hence, the intestine (intestinal stem cells) is extremely vulnerable to radiation injury and is second to bone marrow injury in clinical observation. However, it has been reported that a few weeks before abdominal radiotherapy, $90 \%$ of patients would experience gastrointestinal discomfort ${ }^{[4,5]}$. Western medicine believes that radiation enteritis mainly involves the colon and rectum. Its etiology is related to radiation damage to intestinal tissue epithelium and the change of local vascular permeability as well as intestinal microenvironment, resulting in intestinal mucosal inflammation, bleeding, and flora imbalance, manifesting as abdominal pain, diarrhea, mucus in stools, purulent and bloody stools, etc.

\section{Professor Renting Li's understanding of RE}

$\mathrm{RE}$ is a disease caused by modern medical treatment methods. It is not recorded in ancient medical books, but based on the symptoms, it can be attributed to the syndrome types of "abdominal pain, diarrhea, and bloody stool" in traditional Chinese medicine. Professor Renting Li believes that according to the method of image comparison in traditional Chinese medicine, radiotherapy is similar to the evil of fire and heat in 
the external contraction of the six excesses. Therefore, in irradiation of the human body, the evil of fire and heat is forced by the intestines, which consumes gas, injures body fluid, and burns the veins, manifesting as diarrhea, mucus in stools, as well as purulent and bloody stools. In clinic, acute and chronic RE are often divided by the time of onset. Acute cases often occur during radiotherapy or within half a year after the end of radiotherapy, while chronic cases are mostly due to prolonged symptoms or occur half a year after the end of radiotherapy ${ }^{[6]}$. Its clinical manifestations are abdominal pain, diarrhea, bloody stools, and so on, which affect the quality of life of patients. According to the clinical manifestations and characteristics of $\mathrm{RE}$, Professor Renting Li proposed the use of traditional Chinese medicine retention enema combined with oral traditional Chinese medicine to reduce the symptoms, detoxify, and improve the quality of life.

\subsection{Traditional Chinese medicine retention enema}

Traditional Chinese medicine retention enema is under the category of external treatment in traditional Chinese medicine. Many studies have shown that retention enema allows drugs to directly act on the affected part, thus avoiding various adverse reactions caused by drugs entering the circulation and affecting the metabolism. The external use of drugs can also improve local blood flow and protect the mucosa, so as to stop bleeding and inflammation [7]. Professor Renting Li believes RE is mainly caused by toxic heat, blood stasis, and vein damage; therefore, it is mainly treated by clearing heat, detoxification, and removing blood stasis. The ingredients for the self-made retention enema are as follows: Sargentodoxa, Radix Sophorae Flavescentis, raw sanguisorba, paeonia suffruticosa, Semen Arecae, and Herba Agrimoniae.

\subsection{Oral traditional Chinese Medicine}

Oral traditional Chinese medicine is under the category of internal treatment in traditional Chinese medicine. In consideration of the clinical manifestations, coated tongue, and pulse condition of RE patients, the disease is regarded as a combination of heat and toxin, deficiency in Qi, injury to fluid, and the frenetic movement of blood. The disease is deficiency in reality, and its treatment is mainly based on clearing heat, detoxification, removing blood stasis, and hemostasis. Most patients with recurrent RE, prolonged lesion, and delayed healing have spleen and kidney Qi injury. According to the pathogenesis of chronic RE, Professor Renting Li pointed out that the syndrome differentiation of patients with chronic RE mostly belongs to the weakness of spleen and stomach, deficiency of kidney Qi, and the stasis of heat and poison, which is a mixture of deficiency and excess, but mainly deficiency. The medication used is mainly to strengthen the spleen, warm the middle, and tonify the kidneys.

\section{Case study}

Patient Z, male, 33 years old, was admitted to the first ward of the Affiliated Hospital of Shaanxi University of Chinese Medicine on September 27, 2021, with a complaint of lower abdominal cramps and blood in stools for one day. Five months ago, he was diagnosed with lung cancer with bone metastasis. He received local sacral radiotherapy for 8 times in the First Affiliated Hospital of Xi'an Jiaotong University four months ago. Colonoscopy was performed after the recent admission, and it showed RE. The patient was noted to have a pale tongue with white and thin coating on it along with a thready pulse. He was placed under the category of positive deficiency and blood stasis. The goal of the treatment was to replenish Qi, strengthen his health, remove blood stasis, and stop the bleeding. The medications were as follows: 20 grams of Roasted Radix Astragali seu Hedysari, 12 grams of Ganoderma lucidum, 15 grams of Herba Hedyotis, 15 grams of fried Rhizoma Atractylodis Macrocephalae, 15 grams of Poria cocos, 12 grams of dried orangepeel, and 10 grams of powdered notoginseng to be taken after mixing with water; 12 grams of Caulis Sargentodoxae and 12 grams of raw sanguisorba to be decocted with water, and $300 \mathrm{ml}$ to be taken 
in the morning and evening respectively. Oral traditional Chinese medicine and traditional Chinese medicine retention enema were given to clear heat, detoxify, remove blood stasis, and stop the bleeding. The specific drugs were as follows: 30 grams of Sargentodoxa, 30 grams of Radix Sophorae Flavescentis, 30 grams of raw sanguisorba, 15 grams of paeonia suffruticosa, 30 grams of stir-fried areca, 30 grams of Herba Agrimoniae, and 30 grams of Rhizoma Bletillae to be left for 30 minutes and used twice a day. After two days of treatment, the patient's abdominal pain was relieved, and after three days, patient did not have any bloody stools. He was continued to be treated with the original prescription. After continuous observation for five days, the patient was discharged.

\section{Conclusion and prospects}

Radiotherapy is widely used in the treatment of abdominal and pelvic malignancies with bone metastases. $\mathrm{RE}$ is a possible adverse reaction during and after treatment. Therefore, Professor Renting Li proposed the use of internal and external treatment of traditional Chinese medicine to achieve the purpose of both internal and external treatment and improve the quality of life of patients. However, the external treatment of traditional Chinese medicine is much more than the traditional Chinese medicine retention enema. It has been reported in literatures that acupuncture and massage as well as bean embedding in ear points can also be used for treating RE. It is believed that traditional Chinese medicine can play a greater role in the treatment of RE.

\section{Disclosure statement}

The authors declare that there is no conflict of interest.

\section{References}

[1] Wang J, Yao D, Zheng L, et al., 2019, Expert Consensus on Surgical Management of Chronic Radioactive Intestinal Injury (2019 Edition). Chinese Journal of Practical Surgery, 39(4): 307-311.

[2] Bergonié J, Tribondeau L, 2003, Interpretation of Some Results from Radiotherapy and an Attempt to Determine a Rational Treatment Technique. Yale J Biol Med, 76(4-6): 181-182.

[3] Rubin P, Casarett GW, 1968, Clinical Radiation Pathology as Applied to Curative Radiotherapy. Cancer, 22(4): 767-778. DOI: 10.1002/1097-0142(196810)22:4<767::aid-cncr2820220412>3.0.co;27

[4] Andreyev J, 2005, Gastrointestinal Complications of Pelvic Radiotherapy: Are They of Any Importance. Gut, 54(8): 1051-1054. DOI: 10.1136/gut.2004.062596

[5] Moussa L, Usunier B, Demarquay C, et al., 2016, Bowel Radiation Injury: Complexity of the Pathophysiology and Promises of Cell and Tissue Engineering. Cell Transplant, 25(10): 1723-1746. DOI: 10.3727/096368916X691664

[6] Ministry of Health of the People's Republic of China, 2002, Diagnostic Criteria for Radiation Proctitis, Standards Press of China, Beijing, 2.

[7] Yan J, Lou H, 2016, New Progress in the Prevention and Treatment of Radioactive Enteritis. China Modern Doctor, 54(10): 164-167. 